\title{
MFCA: Matched Filters with Cellular Automata for Retinal Vessel Detection
}

\author{
Oscar Dalmau ${ }^{1}$ and Teresa Alarcon ${ }^{2}$ \\ ${ }^{1}$ Centro de Investigación en Matemáticas, A.C. \\ Jalisco S/N Col. Valenciana, 36240, Guanajuato, Gto. México \\ ${ }^{2}$ Centro Universitario de los Valles \\ Carretera Guadalajara - Ameca Km. 45.5 C.P. 46600. Ameca, Jalisco, México. \\ dalmau@cimat.mx, teresa.alarcon@profesores.valles.udg.mx
}

\begin{abstract}
Blood vessel extraction is an important step for abnormality detection and for obtaining good retinopathy diabetic diagnosis in digital retinal images. The use of filter bank has shown to be a powerful technique for detecting blood vessels. In particular, the Matched Filter is appropriate and efficient for this task and in combination with other methods the blood vessel detection can be improved. We propose a combination of the Matched Filter with a segmentation strategy by using a Cellular Automata. The strategy presented here is very efficient and experimentally yields competitive results compared with others methods of the state of the art.
\end{abstract}

Keywords: Matched filter, cellular automata, blood vessel detection, diabetic retinopathy

\section{Introduction}

Diabetic Retinopathy (DR) is a common complication associated with diabetes. Diabetic Retinopathy is the main cause of blindness among adult people. Then, early Diabetic Retinopathy detection is crucial for decreasing vision loss. Many techniques have been developed in order to detect DR, see for instance [19, 17] and references therein. Most of the automatic DR detection methods also detect normal fundus regions, such as optic disk, fovea, macula and blood vessels. In order to detect retinal lesions, this information (normal fundus regions) is removed due to their similarity, in color and shape appearance, with abnormal retinal areas.

In this work, we propose an automatic blood vessel detection method. Our method has three stages. In the first stage, we enhance the blood vessel regions by using a filter bank. In particular, we use the Matched Filter (MF) reported in [2] due to its efficiency. The resulting image is then thresholded to produce a binary segmentation (second stage). However, thresholding techniques may yield different segmentation results when applying to retinal images with different luminance conditions, or even when using different fundus camera. In order to get good segmentation results, we pay special attention to thresholding techniques

This is a preprint version of a paper published in:

Lecture Notes in Artificial Intelligence, vol. 7094, Springer, 2011.

When citing, please cite the final version published by Springer. 
[15]. Finally, in the third state we improve the previous segmentation by using a Cellular Automata [20,7].

There are several strategies for blood vessel extraction. Some of them are based on: morphological operators [9,21], Neural Networks [13], region growing [12], Gabor Filters [14], second-order Gaussian Filter [3] and Matched Filter $[2,5,22,23]$. Among these methods, the Matched Filter [2] has proven to be very efficient. Most of these methods combine different strategies in order to improve the vessel extraction results. Recently, Zhang et al. [23] proposed a combination of Matched Filter [2] with first-order Gaussian derivative [8]. This method is very efficient and achieves good vessel detection results. The reader can find a study of optimal parameters for obtaining a Matched Filter bank for vessel detection in Ref. [1]. There are two main differences between the proposal in Ref. [23] and our approach. In Ref. [23] Zhang et al. use first-order derivative of Gaussian in order to reduce the problem of strong response of Matched Filter in non-vessel edges. In our algorithm, we pay special attention to the thresholding technique [15]. Instead of using a threshold directly, we relax an automatically obtained threshold value in order to obtain two thresholds: one for the object (vessels) and the other for the background (non-vessels). After applying both thresholds, we are in condition to use a segmentation method. In particular, we use a Cellular Automata [20] as segmentation method. These differences are the principal contributions of this paper in the context of blood vessel detection. We show experimentally that these modifications yield competitive vessel detection results compared with algorithms of the state of the art.

This paper is organized as follows. Section 2 describes our algorithm. Here, we briefly review the Matched Filter and the Cellular Automata algorithm. In Section 3 we present the experimental results. Finally, Section 4 presents our conclusions.

\section{Our Proposal}

The proposed method contains three stages. Firstly, the contrast between vessel and non-vessel is enhanced by applying a filter bank. Then, we relax an automatic threshold value, in order to classify pixels with high confidence of belonging to blood vessel or non-vessel. Pixels with low confidence are finally classified by using a segmentation method.

\section{$2.1 \quad$ Filter Bank}

Many algorithms use filter banks for increasing the contrast of the original image: Gabor Filter, Second order derivative of Gaussian, ... etc. We choose the Matched Filter because is simple and very efficient. The Matched Filter was proposed in [2] and was designed for blood vessel detection in fundus images. The Matched Filter takes into account that the grey level profiles of cross section of blood vessel are similar to a Gaussian function. Although, the intensity profiles can vary in width and intensity, they can be modeled using the Gaussian function: 


$$
f(x, y)=a\left(1-b e^{-\frac{d^{2}}{2 \sigma^{2}}}\right)
$$

where $a, b, \sigma$ are parameters that allows us to model the intensity and width of the vessels, $d$ is the distance from $(x, y)$ to the line passing through the center of the vessel. The reader can find the details in Ref. [2]. Based on the previous function, Eq. (1), the kernel for the Matched filter can simply be designed as follows:

$$
K(x, y)=-e^{-\frac{d^{2}}{2 \sigma^{2}}}
$$

Now, considering a set of orientations $\theta_{i} i=1,2, \cdots, m$ and rotating the kernel in Eq. (2) using these orientations, we obtain the following set of convolution kernels $K_{i}$ at a given scale $\sigma$

$$
\begin{aligned}
\mathbf{t} & =[x, y]^{\prime}, \\
R_{i} & =\left(\begin{array}{cc}
\cos \theta_{i}-\sin \theta_{i} \\
\sin \theta_{i} & \cos \theta_{i}
\end{array}\right), \\
\mathbf{z}_{\mathbf{i}} & =[u, v]^{\prime}=R_{i} \mathbf{t}, \\
K_{i}(x, y) & =-e^{-\frac{u^{2}}{2 \sigma^{2}}, \forall \mathbf{z}_{\mathbf{i}} \in \mathcal{V},}
\end{aligned}
$$

where the neighborhood $\mathcal{V} \stackrel{\text { def }}{=}\left\{(u, v):|u|<3 \sigma,|v|<\frac{L}{2}\right\}, L$ is the length of the neighborhood along the $y$ axis, $\sigma$ represents the scale of the filter and is related with the vessel profile width and $R_{i}$ is a rotation matrix with orientation $\theta_{i}$. Finally, a zero-mean normalization is applied to the Kernel in Eq. (6) to obtain the Matched Filter at orientation $\theta_{i}$ and scale $\sigma$ :

$$
\begin{gathered}
\mu_{i}=\frac{1}{|\mathcal{V}|} \sum_{\mathbf{z}_{\mathbf{i}} \in \mathcal{V}} K_{i}(x, y), \\
\hat{K}_{i}(x, y)=K_{i}(x, y)-\mu_{i}, \forall \mathbf{z}_{\mathbf{i}} \in \mathcal{V},
\end{gathered}
$$

where $|\mathcal{V}|$ is the cardinality of $\mathcal{V}$ and $\mu_{i}$ is the mean value of the Kernel $K_{i}$ in the neighborhood $\mathcal{V}$. In [2] is assumed that the width of the blood vessel is equal to $2 \sigma$.

In summary, the Matched Filter depends on three parameters $\sigma, L$ and $\theta$, i.e., scale, length and orientation respectively. As the scale $\sigma$ is related with the vessel width then, the value of the scales are typically taken in the interval $[1,3], L$ is in $[7,15]$ and $\theta \in\left[0^{0}, 180^{\circ}\right)$. In practice, one uses a discretization of these intervals, for example, $\sigma \in\{1.0,1.1,1.2, \cdots, 2.9,3.0\}, L \in\{7.0,7.1,7.2, \cdots, 14.9,15.0\}$ and $\theta \in\left\{0^{0}, 15^{0}, \cdots, 165^{0}\right\}$. Since we discretize the parameter space, then the parameter search space is finite and not very large. So, an exhaustive search in the parameter space is enough to find the best parameters, i.e., a parameter training step.

In order to build a Matched Filter Bank we must provide a set of scales $\left\{\sigma_{i}\right\}_{i=1,2, \cdots, n}$, a set of lengths $\left\{L_{i}\right\}_{i=1,2, \cdots, n}$ and a set of orientations $\left\{\theta_{i}\right\}_{i=1,2, \cdots, m}$. 
The number of scales and lengths is the same and is equal to $n$, the number of orientation is $m$ and the total number of convolution kernels $\hat{K}_{i}$ of the filter bank is equal to $n * m$. The parameter $L_{i}$ can be selected based on $\sigma_{i}$. If $\sigma_{i}$ is small then $L_{i}$ is relatively small and viceversa [23]. Using this strategy we can eliminate one parameter. The orientations can simply be defined as the number of orientations $(m)$ or the angular resolution $\left(\frac{180^{\circ}}{m}\right)$. According to our experiments and to previous works $[2,1] 12$ orientations, $m=12$, produce good experimental results, i.e., an angular resolution of $15^{0}$. Finally, the blood vessels are enhanced by convolving the fundus image with the set of kernels of the Matched Filter Bank and retaining only the maximum response, denoted here as $M$.

\subsection{Thresholding relaxation}

A natural way for detecting the blood vessels is to binarize the maximum response $M$ of the Matched Filter. However, this simple idea does not always afford good blood vessels extraction. For that reason, different strategies has been proposed for detecting the retinal vessels. For example, Zhang et al. in [23] propose a pixel-wise threshold based on the first-order Gaussian derivative whereas Hoover et al. [5] propose an iterative threshold probing technique in which a sequence of thresholds are applied to the maximum response.

In this work, we propose another strategy. First, we compute an automatic threshold $T$ [15] for segmenting the maximum response of the Matched Filter, Subsection 2.1. In the experiment we use the minimum error thresholding technique proposed by Kittler and Illingworth [6] because it produces the best vessel detection. Then, we relax the previous threshold obtaining two new thresholds $T_{v}, T_{n}$ for vessels and non-vessels respectively. These thresholds are defined in the following way:

$$
\begin{aligned}
& T_{v}=\mu_{v} T, \\
& T_{n}=\mu_{n} T,
\end{aligned}
$$

where $\mu_{n}, \mu_{v}$ are parameters that satisfy $0<\mu_{n}<\mu_{v}$. $T_{v}$ is a threshold above [16] for vessel region, i.e., pixels with maximum response greater than $T_{v}$ have high confidence to belong to the vessel region and are classified as vessel. $T_{n}$ is a threshold below [16] for non-vessel region, i.e., pixels with maximum response less than $T_{n}$ have high confidence to belong to the non-vessel region and are classified as non-vessel. Pixels with maximum response in $\left[T_{n}, T_{v}\right]$ are not classified yet, because they have low confidence to belong to vessel or non-vessel region. The non-classified region is a band between the vessel and non-vessel region. In order to classify these pixels we propose a segmentation stage, see next Section.

\subsection{Segmentation}

Pixels with low Matched Filter maximum response still need to be classified. To this end, we suggest using a segmentation algorithm that allows us to incorporate spatial information and that also takes into account the contrast between 
blood vessels and non-vessels. In particular, in this work we use the "GrowCut" Cellular Automata algorithm $[20,7]$ because it is computationally efficient and experimentally yields good results.

A Cellular Automata is a triplet $A=(S, \mathcal{N}, \delta)$ where $S$ is a non-empty state set, $\mathcal{N}$ is a neighborhood system and $\delta$ is a transition function from the state $t$ to state $t+1$. Let $\mathcal{L}$ be the lattice of the image and $r \in \mathcal{L}$ a pixel of the image. A cell state $S_{r}=\left(l_{r}, \theta_{r}, F_{r}\right)$ is a triplet where $l_{r}$ is the current state, $\theta_{r} \in[0,1]$ is the 'strength' and $F_{r}$ is a feature vector, defined by the image, for details see Refs $[20,7]$. If $\mathcal{N}_{r}$ denotes the set of neighbors of the pixel $r$ and $g: \mathbb{R}^{+} \rightarrow \mathbb{R}^{+}$ is a decreasing function bounded to $[0,1]$, then the transition function of the "GrowCut" is given by the following algorithm:

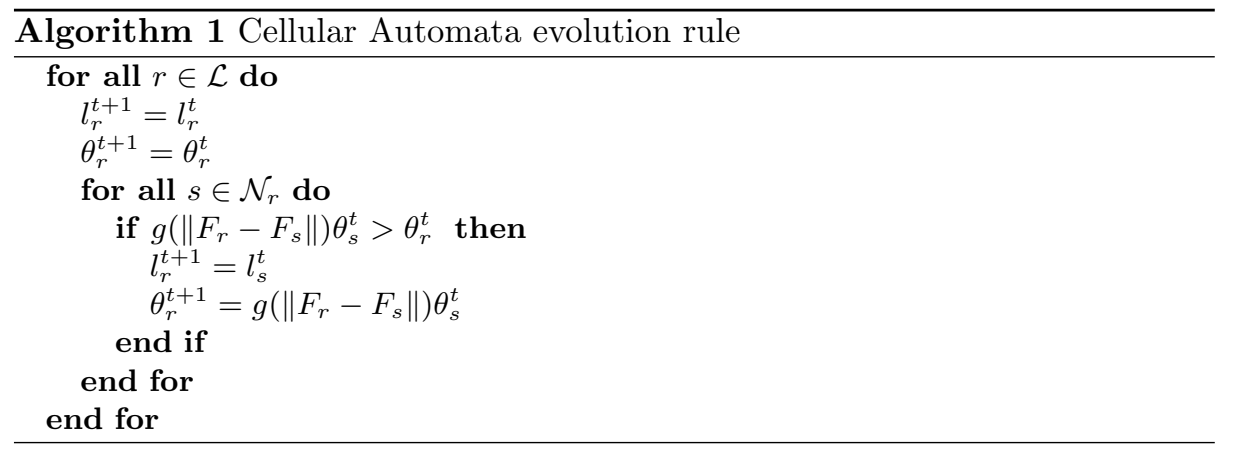

In particular, we use as $\mathcal{N}_{r}$ the set of the first neighbors of r, i.e, $\mathcal{N}_{r}=\{s \in$ $\mathcal{L}:|s-r|=1\}$ and as $g(\cdot)$ the following function:

$$
g(x)=\frac{\gamma}{\gamma+x^{2}},
$$

where $\gamma$ is a positive parameter, in experiments we use $\gamma=10^{-2}$. The feature vector is just the maximum response, i.e., $F_{r}=M_{r}, \forall r \in \mathcal{L}$. The initial state $\forall r \in \mathcal{L}$ is set as follows:

$$
\begin{aligned}
& l_{r}^{0}=\left\{\begin{aligned}
1 & \text { if } M_{r}>T_{v} \\
-1 & \text { if } M_{r}<T_{n} \\
0 & \text { otherwise }
\end{aligned}\right. \\
& \theta_{r}^{0}= \begin{cases}1 & \text { if } M_{r} \notin\left[T_{u}, T_{v}\right] \\
0 & \text { otherwise }\end{cases}
\end{aligned}
$$

\section{Experimental Results}

In this section, we compare our results with other methods of the state of the art. In order to evaluate the algorithm presented here, we test the proposal with 
two databases available online: DRIVE and STARE databases. DRIVE database is available at http://www.isi.uu.nl/Research/Databases /DRIVE/ and STARE database at http://www.ces.clemson.edu/ ahoover/stare/. Both databases are comprised by 20 fundus images, their corresponding masks (or region of interest) and hand-labelled images by two human experts. The image size in the STARE database is $700 \times 605$ pixels and the image size int the DRIVE database is $584 \times 565$ pixels. In both cases, the original images are in color.

In order to compare different detection algorithms $[11,18,23]$ we use the following performance measures: True Positive Rate (TPR), False Positive Rate (FPR) and Accuracy (ACC),

$$
\begin{aligned}
T P R & =\frac{T P}{T P+F N}, \\
F P R & =\frac{F P}{F P+T N}, \\
A C C & =\frac{T P+T N}{T P+F P+F N+T N},
\end{aligned}
$$

where $T P, F P, F N, T N$ are the number of True Positive, False Positive, False Negative and True Negative respectively. The $T P R$ or sensitivity can be interpreted as the capacity of the algorithm for correctly detecting blood vessels. $F P R$ is related with the specificity (FPR $=1-$ specificity). The specificity can be interpreted as the probability of detecting the non-vessel region whereas the accuracy (ACC) is the probability for simultaneously detecting vessel and non-vessel regions.

Table 1. Vessel detection results for the STARE dataset.

\begin{tabular}{lccc}
\hline Method & TPR & FPR & ACC \\
\hline 2nd Human observer & 0.8949 & 0.0610 & 0.9354 \\
Hoover [5] & 0.6751 & 0.0433 & 0.9267 \\
Soares [18] & 0.7165 & 0.0252 & 0.9480 \\
Mendonca [11] & 0.6996 & 0.0270 & 0.9440 \\
MF-FDOG [23] & 0.7177 & 0.0247 & 0.9484 \\
Martinez-Perez [10] & 0.7506 & 0.0431 & 0.9410 \\
MF-MET & $\mathbf{0 . 7 3 8 0}$ & $\mathbf{0 . 0 6 0 4}$ & $\mathbf{0 . 9 1 8 4}$ \\
MFCA & $\mathbf{0 . 7 6 0 6}$ & $\mathbf{0 . 0 5 9 9}$ & $\mathbf{0 . 9 2 1 2}$ \\
\hline
\end{tabular}

As it has been noted before in different works [18] and references therein, the green channel of retinal images shows the best vessel/background contrast while the red and blue channels are very noisy and have a lower contrast. Therefore, we use the image green channel in all experiments. Similar to the previous work in Ref. [23] we use as ground truth the hand-labelled image by the first expert.

In the experiments we use the following parameters: the angular resolution is $15^{\circ}, \sigma=1.4$ (scale), $L=11$ (length), $\mu_{v}=1.05$ (threshold factor 
for the blood vessels) and for the threshold factor for the non-vessel region we use $\mu_{n}=0.84$ for the DRIVE database and $\mu_{n}=0.92$ for the STARE database, the number of iterations of the Cellular Automata is 10, see Section 2. All these parameters were found by minimizing the segmentation error over the discrete parameter space defined by $\sigma \in\{1.0,1.1,1.2, \cdots, 3.0\}, L \in$ $\{7.0,7.1,7.2, \cdots, 15.0\}, m \in\{3,4,5,6,8,9,10,12,15\}, \mu_{n} \in\{0.8,0.81, \cdots, 0.99\}$ and $\mu_{v} \in\{1.01,1.02, \cdots, 1.2\}$.

Table 2. Vessel detection results for the DRIVE dataset.

\begin{tabular}{lccc}
\hline Method & TPR & FPR & ACC \\
\hline 2nd Human observer & 0.7761 & 0.0275 & 0.9473 \\
Soares $[18]$ & 0.7283 & 0.0212 & 0.9466 \\
Mendonca [11] & 0.7344 & 0.0236 & 0.9452 \\
MF-FDOG [23] & 0.7120 & 0.0276 & 0.9382 \\
Martinez-Perez [10] & 0.7246 & 0.0345 & 0.9344 \\
MF-MET & $\mathbf{0 . 6 9 9 2}$ & $\mathbf{0 . 0 4 5 9}$ & $\mathbf{0 . 9 1 9 9}$ \\
MFCA & $\mathbf{0 . 7 6 2 0}$ & $\mathbf{0 . 0 5 9 2}$ & $\mathbf{0 . 9 1 6 8}$ \\
\hline
\end{tabular}

In some works, the authors give no detail about the thresholding method, for example in [23]. However, vessels detection can be very sensitive to the thresholding method. We experiment with several global thresholding techniques: Otsu, entropy, moments, concavity, minimum error, intermeans, intermodes,..., etc [15, 4]. For this experiment we use all the thresholding techniques provided by the HistThresh toolbox for MATLAB available at http://www.cs.tut.fi/ ant/ histthresh/. Among them, the minimum error thresholding technique, proposed by Kittler and Illingworth [6] is the thresholding method that produces the best vessel detection. Therefore, in the experiments we use the minimum error thresholding technique for computing $T$, Subsection 2.2 .

Tables 1-2 show the results obtained with our proposal and other methods of the state of the art. We include the result obtained after applying the minimum error thresholding technique, Matched Filter with Minimum Error Thresholding (MF-MET), i.e., the first two steps of Section 2. The purpose of including the results of MF-MET is to prove that the Cellular Automata improves the segmentation results. The experimental results of the algorithms of the state of the art are taken from Zhang et al. [23], recently published, see also Refs. [5, 18, 11, 10, 23]. As we can see in the first column of tables 1-2, the method proposed here, MFCA, presents the best vessel detection results. Second and third columns of tables 1-2 show that our proposal yields competitive results compared with methods of the state of the art for detecting vessels and non-vessel regions. Note that the sensitivity of the method by using Cellular Automata has increased the True Positive Rate (TPR) vessel detection percentage; nevertheless, the False Positive Rate (FPR) has increased as well. The accuracy ACC therefore dimin- 


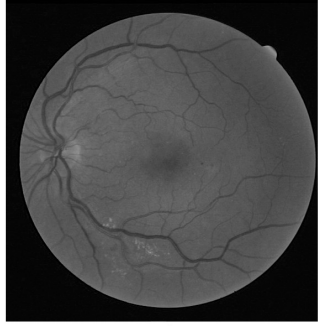

a)

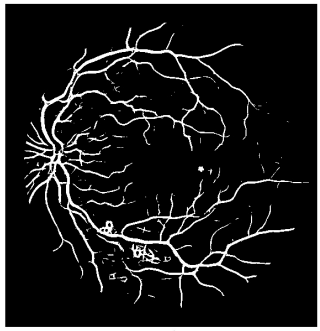

c)
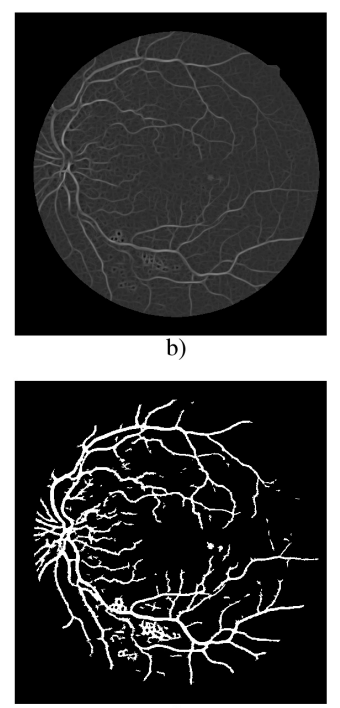

d)

Fig. 1. '03-test' image of the DRIVE database. a) Green channel, b) Maximum response to Matched Filter, c) Segmentation using MF-MET, d) Segmentation using MFCA.

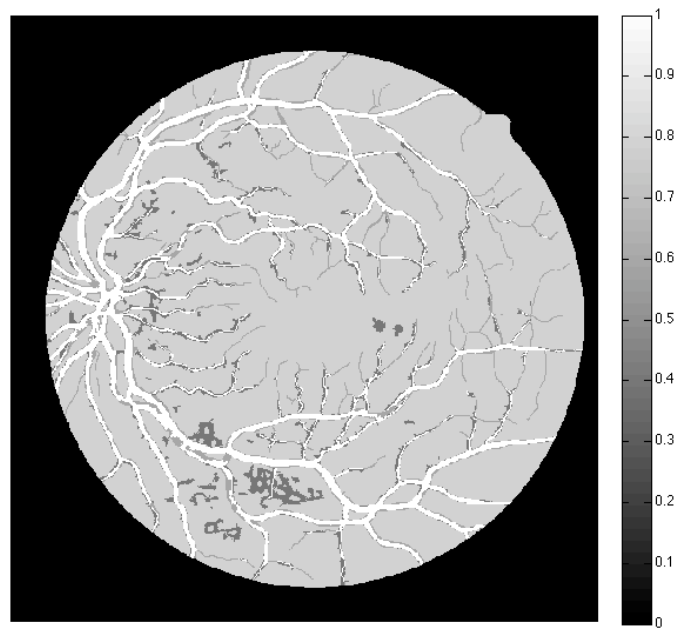

Fig. 2. Confusion image corresponding to '03-test' image of the DRIVE database, see Fig. 1 a). True Positive: gray level image equal to 1.0, True Negative: gray level image equal to 0.8, False Negative: gray level image equal to 0.6 and False Positive: gray level image equal to 0.4 . 
ishes a little bit. Hence, there is a trade off between TPR and FPR. On the other hand, the consuming time of the proposed method is low. The running time average per image in the STARE database by using the MF-MET algorithm is 0.4 seconds and the running time average of the MFCA algorithm is 1.0 second. The implementation is in Matlab 7.10 and we run the script in a Core i7 2.2 $\mathrm{GHz}$ with $4 \mathrm{~GB}$ of RAM.

Figures 1-2 illustrate the result obtained by using the MFCA algorithm to the '03-test' image of the DRIVE database. The '03-test' image was randomly selected from the DRIVE database for illustration purposes. Figure 1 panel a) shows the green channel of the original image, panel b) depicts the maximum response of the Matched Filter Bank, panel c) is the segmentation after applying the minimum error thresholding and finally, panel d) shows the blood vessel detection of the MFCA algorithm. Figures 2 depicts the confusion image, defined here as the image of TP, TN, FP and FN results. This representation is very important because it allows us to detect the regions where the algorithm obtains good segmentation results and where the algorithm is wrong. The gray levels 1.0 and 0.8 correspond to regions where the MFCA algorithm correctly detects the blood vessels and non-vessels regions respectively, i.e., TP and TN results. The gray levels 0.6 and 0.4 correspond to regions where the MFCA algorithm is wrong, i.e., FP and FN results. From this figure, we can see that the main limitation of our proposal is for detecting thin vessels. A possible solution for this problem is to use different scales in the MF bank, similar to the previous work by Zhang et al. [23]. Another problem is that our algorithm detects some retinal lesions as vessels. This is due to the MF bank has high response in regions with high contrast, i.e., hard exudates, see Fig. 1 panels a) and b). We note that unlike other algorithms, our method does not pre process or post process the image. Therefore, we can improve our results by making a pre-processing of the image in order to eliminate regions with high contrast, because it is wellknown that regions with high contrast correspond to exudates or the optic disc. A simple alternative is to remove the high contrast regions using a thresholding technique, and a more sophisticated alternative could be to design filters that have low response in regions with high contrast.

\section{Conclusion}

In this paper we present a three-stage algorithm for automatic blood vessel extraction. We show experimentally that our proposal presents the best vessel detection results compared with methods of the state of the art. The proposed algorithm is simple, computationally efficient and easy to implement. The nonvessel region extraction and the simultaneous detection of vessel and non-vessel regions are competitive with the methods of the state of the art. The main problem of our method is for detecting thin vessels. Another difficult is that our algorithm detects some lesions as vessels. One way to solve this problem is to make a pre-processing or a post-processing to eliminate areas of high contrast. Despite of our strategy was proposed in the context of retinal images for detecting 
blood vessels it is not limited to this kind of images. There are several image processing tasks in which this algorithm can be used, for example, for road detection in satellite imagery and for carbon nanotube detection in order to study properties of nanomaterials.

Acknowledgments. The authors wish to thank Dra. Graciela Farias and Dr. Antonio Costilla for their advices and the constant encouragement for this work. This research was supported in part by CONACYT (Grant 105657).

\section{References}

1. Al-Rawi, M., Qutaishat, M., Arrar, M.: An improved matched filter for blood vessel detection of digital retinal images. Computers in Biology and Medicine 37, 262-267 (February 2007)

2. Chaudhuri, S., Chatterjee, S., Katz, N., Nelson, M., Goldbaum, M.: Detection of blood vessels in retinal images using two-dimensional matched filters. IEEE Transactions on Medical Imaging 8(3), 263-269 (1989)

3. Gang, L., Chutatape, O., Krishnan, S.M.: Detection and measurement of retinal vessels in fundus images using amplitude modified second-order Gaussian filter. IEEE Transactions on Biomedical Engineering 49(2), 168-172 (2002)

4. Gorman, L.O., Sammon, M.J., Seul, M.: Practical Algorithms for Image Analysis: Description, Examples, Programs and Projects. Cambridge University, 32 Avenue of the Americas, New York (2008)

5. Hoover, A., Kouznetsova, V., Goldbaum, M.: Locating blood vessels in retinal images by piecewise threshold probing of a matched filter response. IEEE Transactions on Medical Imaging 19, 203-210 (2000)

6. Kittler, J., Illingworth, J.: Minimum error thresholding. Pattern Recognition 19, 41-47 (January 1986)

7. Konushin, V., Vezhnevets, V.: Interactive image colorization and recoloring based on coupled map lattices. In: In Graphicon. pp. 231-234 (2006)

8. Li, Q., Zhang, L., You, J., Zhang, D., Bhattacharya, P.: Dark line detection with line width extraction. In: IEEE International Conference on Image Processing, ICIP'08. pp. 621-624 (2008)

9. Luo, Z., Liu, Z., Li, J.: Micro-blood vessel detection using k-means clustering and morphological thinning. In: Proceedings of the 8th International Conference on Advances in Neural Networks - Volume Part III. pp. 348-354. ISNN'11, SpringerVerlag, Berlin, Heidelberg (2011)

10. Martinez-Perez, M., Hughes, A., Thom, S., Bharath, A.A., Parker, K.: Segmentation of blood vessels from red-free and fluorescein retinal images. Medical Image Analysis 11, 47-61 (2007)

11. Mendonca, A., Campilho, A.: Segmentation of retinal blood vessels by combining the detection of centerlines and morphological reconstruction. IEEE Transactions on Medical Imaging 25(9), 1200 -1213 (sept 2006)

12. Palomera-Pérez, M.A., Martínez-Pérez, M.E., Bentez-Pérez, H., Ortega-Arjona, J.L.: Parallel multiscale feature extraction and region growing: application in retinal blood vessel detection. IEEE Transactions on Information Technology in Biomedicine pp. 500-506 (2010) 
13. Perfetti, R., Ricci, E., Casali, D., Costantini, G.: Cellular Neural Networks with Virtual Template Expansion for Retinal Vessel Segmentation. IEEE Transactions on Circuits and Systems II: Analog and Digital Signal Processing 54, 141-145 (2007)

14. Rangayyan, R.M., Ayres, F.J., Oloumi, F., Oloumi, F., Eshghzadeh-Zanjani, P.: Detection of Blood Vessels in the Retina Using Gabor Filters. J. Electronic Imaging 17(2), 023018 (2008)

15. Sezgin, M., Sankur, B.: Survey over image thresholding techniques and quantitative performance evaluation. Journal of Electronic Imaging 13(1), 146-168 (2004)

16. Shapiro, L.G., Stockman, G.C.: Computer Vision. Prentice Hall, Upper Saddle River, New Jersey (2001)

17. Singh, N., Tripathi, R.C.: Automated early detection of diabetic retinopathy using image analysis techniques. International Journal of Computer Applications 8(2), 18-23 (October 2010), published By Foundation of Computer Science

18. Soares, J.V.B., Le, J.J.G., Cesar, R.M., Jelinek, H.F., Cree, M.J., Member, S.: Retinal vessel segmentation using the 2-D Gabor wavelet and supervised classification. IEEE Transactions on Medical Imaging 25, 1214-1222 (2006)

19. Usher, D., Dumskyj, M., Himaga, M., Williamson, T.H., Nussey, S., Boyce, J.: Automated detection of diabetic retinopathy in digital retinal images: a tool for diabetic retinopathy screening. Diabetic Medicine a Journal of the British Diabetic Association 21(1), 84-90 (2004)

20. Vezhnevets, V., Konouchine, V.: "GrowCut" - Interactive Multi-Label N-D Image Segmentation By Cellular Automata. In: Graphicon-2005. pp. 150-156. Novosibirsk Akademgorodok, Russia (2005)

21. Zana, F., claude Klein, J.: Segmentation of vessel-like patterns using mathematical morphology and curvature evaluation. IEEE Transactions on Image Processing 10, 1010-1019 (2001)

22. Zhang, B., Li, Q., Zhang, L., You, J., Karray, F.: Retinal vessel centerline extraction using multiscale matched filter and sparse representation-based classifier. In: Second International Conference on Medical Biometrics, ICMB 2010. pp. 181-190 (2010)

23. Zhang, B., Zhang, L., Zhang, L., Karray, F.: Retinal vessel extraction by matched filter with first-order derivative of Gaussian. Computers in Biology and Medicine 40, 438-445 (April 2010) 